\title{
Power Optimization Techniques in Wireless Sensor Networks
}

\author{
Veeresh M. Hiremath and Dr.G.A. Bidkar
}

\begin{abstract}
Wireless sensor network (WSN) is the network of small size, low cost, low weight sensors which are capable of sensing, transmitting, receiving and processing. The microcontroller inside the sensor node processes the sensed information and forwards it to the neighboring node. The information is den forwarded to the sink node through multiple hops. Nodes which are in the vicinity of the sink node will be receiving and forwarding the information from all other nodes. The power consumed in transmitting and receiving the data is much more than the power consumed by the microcontroller for processing the information. Because of this fact the nodes which are in the vicinity of the sink node deplete their energy faster as compared to the other nodes. In clustering the distance between the node and the sink node is reduced thereby reducing the power consumption. By properly selecting the cluster head, the power consumed in transmitting and receiving can be minimized.
\end{abstract}

Keywords--- Cluster Head, Clustering, Mobile Base Station, Sink Node

\section{INTRODUCTION}

$\mathrm{W}$ $\mathrm{SN}$ is a collection small size, low cost, low weight sensors which sense different parameters of a particular system and sends it to the sink node wirelessly. WSNs find applications in various areas such as health, forest, environment, military and agriculture.

In Medical field, sensors are used to monitor the different physiological parameters of a patient such as body temperature, blood pressure, heart beat and forward this information to a remote control station wirelessly [1]. The doctor or the nurse, sitting at a far place can take necessary measures depending on the health condition of the patient. WSN technology is very fast growing and various new applications are emerging in the near future. Researchers are going on improving the performance of WSN such as optimization, increasing the transmission range and the type of connection topologies[2].

In this paper, a survey is made on different methods of optimizing the power.

Veeresh M. Hiremath, Research Scholar, Dept of ECE, S.D.M,C.E.T., Karnataka. India.E-mail:vireshblh@gmail.com

Dr.G.A. Bidkar, Professor \& Head, Dept of ECE, S.D.M,C.E.T., Karnataka, India. E-mail:gabidkar@rediffmail.com

DOI:10.9756/BIJRCE.8207

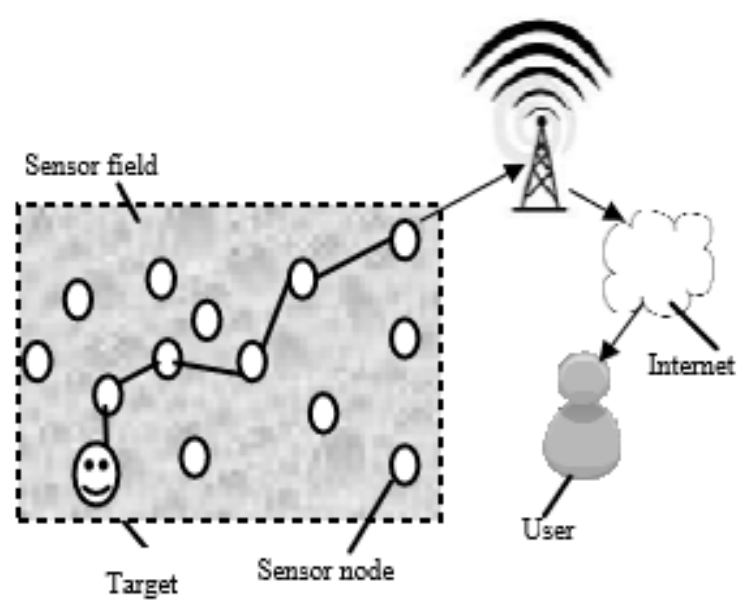

Figure 1: Architecture of Wireless Sensor Networks

The rest of this paper is organized as follows. In section 2, we present a general background on WSN concepts like power management and power conservation. Section 3 describes power optimization by clustering method and different method of selecting the cluster heads. Section 4 explains how power can be saved by making the base station mobile.

\section{BACKGROUND}

As WSN is an infrastructure-less network, each node has finite power capabilities. The battery is limited and manual power replenishment is not possible. The efficient management of energy is a critical issue in wireless sensor networks. Main causes of battery depletion are:

1. Packet Collision: In case of packet collision the packet has to be retransmitted. This leads to additional energy consumption

2. Idle Listening: Sensor nodes are always ON and ready to receive any incoming packet.

3. Overhearing: Nodes receive data which is intended for other nodes.

4. Multi-Hopping: Nodes which are closer to the base station get depleted faster. This is because they do the additional task of forwarding packets from other nodes in addition to their own.

The life span of a sensor network depends on the life span of sensor node which in turn depends on the power consumption at each sensor node. A more efficient power management will provide a longer network lifetime[3]. A variety of dynamic power management techniques have been proposed to reduce the power consumption in sensor nodes and in general battery-powered embedded systems[4,5] by selectively shutting down components. Much work has been done exploiting the sleep state and active power management. 
For different network topologies, communication protocols are devised. A frame work has been proposed for the routing problem of WSNs called Sensor system for Hierarchical Information gathering through Virtual triangular Areas (SHIVA) [6]. Some approaches are presented to move the location of the gateway (sink) node for optimized communication energy and timeliness address the issue of energy depletion in gateway nodes by presenting approaches. One of the proposed solutions is to move the gateway to an area where the volume of real-time data is high, and thus balance the traffic load among the nodes in that area. The basic issues like when and where the gateway should be relocated and how the traffic should be managed during its movement are also tackled [4].

Integer linear Programming model is used to determine locations of multiple sinks in the network. A new mobile base stations could be deployed to deal with the energy depletion due to gateway nodes. The locations of the base stations are periodically changed [7].

\section{CLUSTERING}

If a large geographical area is to be considered for sensing with a stationary base station, sensor nodes have to send the data for a long distance through multiple hops. As the distance and number of hops increase, the power consumption also increases.

If the area is divided into multiple clusters, and one base station or cluster head is nominated for each of the cluster, the distance between the cluster head and other nodes decreases and the number of hops taken by data packet to reach the cluster head also decreases. This saves large amount of power.

In selecting the cluster head, man issues are to be considered such as residual energy of cluster head, residual energy of other sensor nodes.

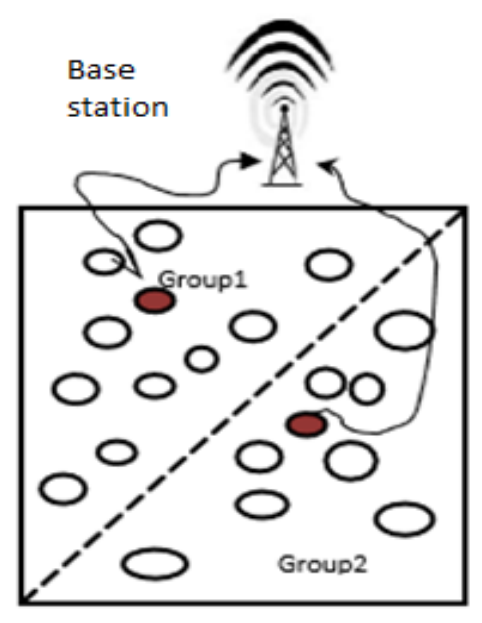

Figure 2: Clustering Method for Power Optimization

LEACH (Low Energy Adaptive Clustering Hierarchy) is an efficient cluster based protocol. This reduces the number of hops of data packet for reaching the base station.

The LEACH protocol works in multiple rounds. Initially when clusters are being selected, each node decides whether to become the cluster head for the current round or not. Every node chooses a random number $\mathrm{n}$ between 0 and 1 . If the number selected is less than the threshold, the node under consideration becomes the cluster head for the current round. The threshold value is computed as

$$
T(n)=\left\{\begin{array}{cc}
p & \text { if } n \in G \\
1-p * r \text { mod } \frac{1}{p} & \text { otherwise } \\
0 & \text { other }
\end{array}\right.
$$

Where $p$ is the desired percentage of cluster heads, $r$ is the current round and $G$ is the set of nodes that have not been the cluster heads for last $1 / p$ number of rounds.

\section{Mobile Multiple Base Stations}

By using the clustering method power consumption can be minimized. Every cluster will be having a cluster head. This head collects information from all the sensors in that cluster. Cluster heads are selected by LEACH protocol. But the cluster heads are stationary, they are not mobile. If cluster heads or the base stations are made to move inside the cluster then power consumption will be still minimized. This is because; the nodes will send data to the base station whenever the base station moves in vicinity to them. The path is to be selected in such a way that, on the path the base station covers as maximum number of nodes as possible.

\section{CONCLUSION}

In this paper, two methods are discussed for optimizing the power in wireless sensor networks. By deploying multiple base stations and making them mobile, power can be optimized to the maximum extent but it puts overhead on controlling and determining the path in which the base station needs to be moved.

\section{ACKNOWLEDGMENT}

I sincerely thank my respected guide, Dr. G. A. Bidkar for encouraging and guiding me in the preparation of this paper. I thank my fellow colleagues and friends for helping me in preparing this paper. I also thank all the technical staff of the research centre for providing me the facilities for preparing this paper.

\section{REFERENCES}

[1] K. Sohraby, D. Minoli and T. Znati, "Wireless Sensor Network, Technology, Protocols, and Applications”, John Wiley \& Sons, New Jersey, Canada, Pp.10-23, 2007.

[2] A. Esmailpour, M. Alfaraj, J. Alfaraj and G. Kokabian, "Energy Conservation for Wireless Sensor Networks Using Solar Rechargeable Power Source", ASEE Northeast Section Conference, Norwich University, Pp.14-16, 2013.

[3] T. Mantoro, H.S. Ali, W. Usino and M.M. Kadhum, "Energy Efficiency Mechanisms Using Mobile Node in Wireless Sensor Networks", International Conference on Networked Digital Technologies, Pp.536-550, 2012.

[4] A. Sinha and A. Chandrakasan, "Dynamic Power Management in Wireless Sensor Networks", IEEE Design and Test of Computers, Vol.18, No.2, Pp.62-74, 2001.

[5] E.Y. Chung, L.B. Enini and G.D. Micheli, "Dynamic Power Management Using Adaptive Learning Tree”, International Conference on Computer-Aided Design (ICCAD), Pp. 274-279, 1999.

[6] D. Prasad Bavirisetti and N. Prasad Mandru and Sibaram Khara, "Optimal power management in wireless sensor networks for enhanced life time”, Vol.3, No.4, 2012. 